\title{
Electron beam diagnostics for a superconducting radio frequency photoelectron injector
}

\author{
Thorsten Kamps, ${ }^{1, a)}$ Andre Arnold, ${ }^{2}$ Daniel Boehlick, ${ }^{1}$ Marc Dirsat, ${ }^{1}$ Guido Klemz, ${ }^{3}$ \\ Dirk Lipka, ${ }^{1, b)}$ Torsten Quast, ${ }_{1}^{1}$ Jeniffa Rudolph, ${ }^{1}$ Mario Schenk, ${ }^{1}$ Friedrich Staufenbiel, ${ }^{2, c)}$ \\ Jochen Teichert, ${ }^{2}$ and Ingo Will ${ }^{3}$ \\ ${ }^{1}$ Berliner Elektronenspeicherring-Gesellschaft für Synchrotronstrahlung, BESSY Albert-Einstein-Strasse 15, \\ 12489 Berlin, Germany \\ ${ }^{2}$ Forschungszentrum Dresden-Rossendorf, FZD, Bautzner Landstrasse 128, 01328 Dresden, Germany \\ ${ }^{3}$ Max-Born-Institut Berlin, MBI, Max-Born-Straße 2 A, 12489 Berlin, Germany
}

(Received 15 April 2008; accepted 7 July 2008; published online 2 September 2008)

\begin{abstract}
A superconducting radio frequency (SRF) photoelectron injector is currently under construction by a collaboration of BESSY, DESY, FZD, and MBI. The project aims at the design and setup of a continuous-wave SRF injector including a diagnostics beamline for the ELBE free electron laser (FEL) and to address R\&D issues on low emittance injectors for future light sources such as the BESSY FEL. Of critical importance for the injector performance is the control of the electron beam parameters. For this reason a compact diagnostics beamline is under development, serving a multitude of operation settings. In this paper the layout and the rationale of the diagnostics beamline are described. Furthermore detailed information on specific components is given, together with results from laboratory tests and data taking. (c) 2008 American Institute of Physics.
\end{abstract}

[DOI: $10.1063 / 1.2964929]$

\section{MOTIVATION}

Future free electron laser (FEL) light sources such as the proposed BESSY FEL (Ref. 1) and the ELBE facility ${ }^{2}$ operate with superconducting radio frequency (SRF) for electron acceleration to enable continuous-wave (cw) operation generating flexible bunch patterns.

At the ELBE facility, electron bunches are generated in a thermionic injector with a normalized emittance of $10 \mathrm{~mm} \mathrm{mrad}$ at an $80 \mathrm{pC}$ bunch charge. In order to increase the brightness of the FEL, an upgrade program was started and options were investigated to improve the linac performance. Radio frequency (rf) photoinjectors offer excellent performance characteristics for application at FELs. Strong rf accelerating fields together with emittance compensating solenoidal fields deliver high bunch charges at low normalized emittances. While normal conducting radio frequency (NCRF) photoinjectors have been run $^{3}$ and are planned to operate $^{4}$ at high duty cycles and low emittances, operation of an injector at high gradients and at a $100 \%$ duty cycle is possible using SRF technology. Hence the SRF photoinjector combines the advantages of photoassisted production of short electron pulses and high-gradient, low-loss cw operation of a superconducting cavity. The main challenges are

- the design of the superconducting cavity with a suitable cathode insertion,

\footnotetext{
${ }^{\text {a) }}$ Author to whom correspondence should be addressed. Electronic mail: kamps@bessy.de.

${ }^{b}$ Present address: Deutsches Elektronen-Synchrotron, DESY, Notkestrasse 85, 22607 Hamburg, Germany.

${ }^{c}$ Present address: Hahn Meitner Institut, HMI, Glienicker Straße 100, 14109 Berlin, Germany.
}

- the choice of the cathode material,

- the risk of contamination of the cavity with the cathode material, and

- a method to control the transverse emittance of the electron beam.

The emittance compensation scheme, which makes use of the interplay between the fields of the accelerating rf wave and a solenoidal field, now commonly used in NCRF guns, cannot be used in a SRF gun without modifications. When a superconducting material is placed in direct vicinity of strong magnetic fields, magnetic flux lines might be trapped. In the case of a SRF gun cavity this leads to a vast enlargement in the surface resistance, thus beating down the quality factor and impeding operation at the desired accelerating gradients. Numerical investigations ${ }^{5}$ show, however, that the split photoinjector concept can be applied to the SRF gun case. Due to the large distance required between the solenoid and the gun, the space-charge induced blowup is severe. According to the invariant envelope matching criterion, the location of the booster moves further away from the gun and the accelerating field in the booster is relatively low, rendering the whole scheme not very efficient. However, a solenoid is included in the SRF injector beamline to test these issues, but it will be mainly used to focus the divergent beam from the gun. Further, other designs for a SRF electron injector ${ }^{6}$ also use a solenoid in close proximity to the gun. Other schemes to achieve low emittances, such as rf focusing ${ }^{7}$ with a retracted cathode and a shaped cathode visor, will be tried within the framework of this project.

The SRF gun collaboration of BESSY, DESY, FZR, and MBI sets out to tackle all these issues. The target of the collaboration is to set up a SRF gun ${ }^{8}$ together with a diag- 


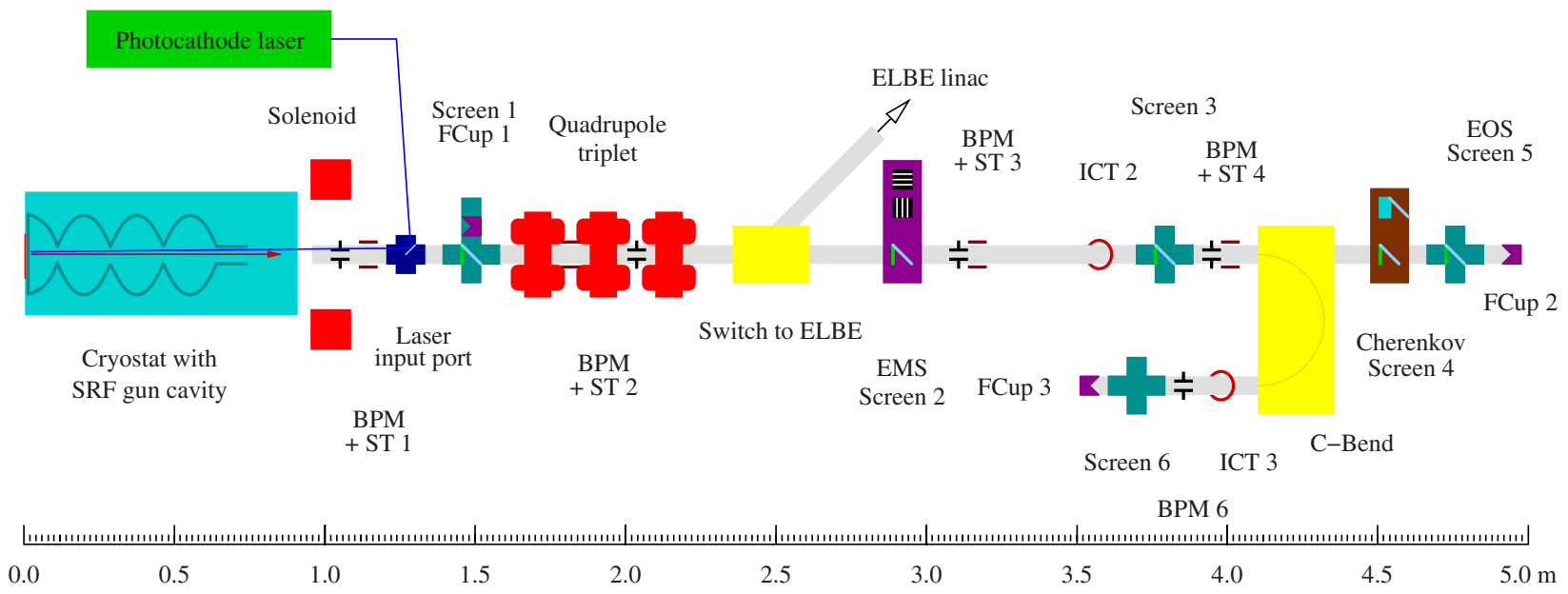

\begin{tabular}{|c|c|c|c|}
\hline \multicolumn{4}{|l|}{ Legend } \\
\hline$\stackrel{\perp}{\mathbf{T}} \mathrm{BPM}$ & Beam Position Monitor & $\bigcirc \mathrm{ICT}$ & Integrating Current Transformer \\
\hline ST & Steering coil & $\equiv$ EMS & Emittance measurement system \\
\hline FCup & Faraday cup & Cherenkov & Bunch length monitor \\
\hline Screen & Viewscreen station & & \\
\hline
\end{tabular}

FIG. 1. (Color online) General setup of the SRF gun and diagnostics beamline.

nostics beamline to serve as a test facility for future SRF injectors. The task of the diagnostics beamline is to ensure safe operation of the injector, check the theoretical model for the injector by measurements, and find an optimum working point for the setup of the injector parameters.

\section{EXPERIMENTAL SETUP}

The setup for the photoinjector consists of the SRF gun and the diagnostics beamline, as depicted in Fig. 1. The electrons are generated by a pulsed laser beam impinging on the photocathode. The laser is designed to operate at a $500 \mathrm{kHz}$ repetition rate delivering pulses of $16 \mathrm{ps}$ full width at half maximum (FWHM) length with a $1 \mu \mathrm{J}$ pulse energy at a $263 \mathrm{~nm}$ wavelength. ${ }^{9}$ The repetition rate of the laser can be reduced for alignment and beam diagnostics measurements. This laser serves two operation modes at high bunch charges. A second laser is under development for operation of the injector at a higher repetition rate $(13 \mathrm{MHz})$ with a reduced bunch charge of $77 \mathrm{pC}$. The three main operation modes of the injector are summarized in Table I. The photocathode is

TABLE I. Design beam parameters of the three main operation modes of the SRF injector: for the ELBE FEL replacing the thermionic gun (ELBE), at high bunch charge (HC), and BESSY FEL (BFEL).

\begin{tabular}{lccc}
\hline \hline & ELBE & HC & BFEL \\
\hline rf & & $1.3 \mathrm{GHz}$ \\
Beam energy & & $9.5 \mathrm{MeV}$ \\
Operation & \multicolumn{3}{c}{} \\
Drive laser & \multicolumn{3}{c}{$263 \mathrm{~nm}$} \\
Photocathode & 5 & $\mathrm{Cs}_{2} \mathrm{Te}$ \\
Pulse length FWHM $(\mathrm{ps})$ & $13 \mathrm{MHz}$ & $500 \mathrm{kHz}$ & $16 \mathrm{kHz}$ \\
Repetition rate & $77 \mathrm{pC}$ & $1 \mathrm{nC}$ & $2.5 \mathrm{nC}$ \\
Bunch charge & 1.5 & 2.5 & 3 \\
Transverse emittance $(\mu \mathrm{m})$ & & & \\
\hline \hline
\end{tabular}

made of $\mathrm{Cs}_{2}$ Te with a quantum efficiency of better than $1 \%$. This cathode is placed at the entrance of a $3 \frac{1}{2}$ cell cavity structure with a rf of $1.3 \mathrm{GHz}$. During the commissioning of the SRF cavity, the gun was operated with a copper cathode, which has a quantum efficiency three orders of magnitude lower than that of $\mathrm{Cs}_{2} \mathrm{Te}$. The motivation for this is to separate the different commissioning stages and be able to fully test one subsystem after the other without mixing too many difficulties. The axis peak field of the accelerating mode is $E_{\text {peak }}=50 \mathrm{MV} / \mathrm{m}$ (design value). The current cavity is able to produce an accelerating field of $E_{\text {acc }}=6 \mathrm{MV} / \mathrm{m}$ due to problems encountered during the cleaning process. ${ }^{8}$ The cavity structure is embedded in a liquid helium tank. At the exit of the SRF gun the electron beam is focused with a solenoid magnet. After that, the electrons pass the transfer section, preparing the beam parameters for injection into the ELBE linac. Behind this section the diagnostics beamline is located, and the beam parameters will be measured.

\section{COMMISSIONING AND OPERATION}

The operation time of the photoinjector can be divided into its commissioning, characterization, and optimization phases. For each phase, beam diagnostics is necessary to facilitate the required beam measurements.

\section{A. Commissioning procedures}

During the commissioning phase the injector will be checked and set up for measurements. Some injector parameters, such as the laser position on the cathode and thus the emission area and the position of the solenoid with respect to the electron beam path, cannot be controlled directly with sufficient accuracy; therefore beam based techniques need to be applied. This requires a minimum set of beam diagnostics tools at the gun exit to measure the bunch charge and the transverse beam position for bunches at low charges and low 
energies. For these measurements a Faraday cup and a scintillating screen (viewscreen) are mounted in a vacuum port directly after the solenoid and laser input port. The distance of the viewscreen is right for imaging of the cathode surface illuminated by the laser beam. During the commissioning phase, the main purpose of the first viewscreen is to observe movement of the center of gravity of the transverse charge distribution. Later, during routine operation, orbit observation will be performed by a set of beam position monitors (BPMs), which are spread along the beamline. The aim of the beam based laser alignment procedure is to move the laser beam on the cathode area to a position where the emitted electrons are not deflected by the rf field of the gun cavity. At each laser position the laser phase relative to the rf phase is scanned. This procedure will be iterated by beam based alignment of the solenoid, where the solenoidal field is varied and the movement of the beam on the first viewscreen is observed, until the beam passes both fields steering-free. For safe operation of the injector, full transmission from the electron source to the beam dump needs to be ensured. Integrating current transformers (ICTs) capable of monitoring small-scale charge fluctuations are put into the beamline to quickly discover beam losses. In addition to the ICTs, the BPM system is able to monitor charge fluctuations. One cause for charge fluctuations is intensity variations in the laser pulses. These intensity variations can be counteracted by controlling the laser intensity and monitoring the produced charge.

\section{B. Beam characterization}

Full reconstruction of the six-dimensional phase space is required for proper modeling of the injector beam dynamics and to investigate different schemes for optimum emittance production. The scope of application for this phase of the SRF injector project is focused on the projected beam parameters. For a better understanding and modeling, slice measurements are necessary. These will be addressed in the next stage of the SRF injector project.

In order to characterize the performance of the photoinjector, the following beam parameters need to be considered:

- The energy distribution of the beam-the kinetic energy of the electrons and the energy spread. The beam energy will vary between a few and $9.5 \mathrm{MeV}$. The minimum energy spread as expected from simulations will be $36 \mathrm{keV}$ for the low charge operation mode.

- The total beam intensity, together with the time structure. The bunch charge can vary between a few picocoulombs during operation with a copper cathode and $2.5 \mathrm{nC}$ for nominal operation in the BESSY FEL mode.

- The optical properties, which can be described in terms of the transverse beam emittance. The normalized beam emittance is expected to vary between 1 and $10 \mathrm{~mm}$ mrad.

The optical properties are dependent on the intensity and energy distribution of the beam. Therefore all parameters need to be analyzed.

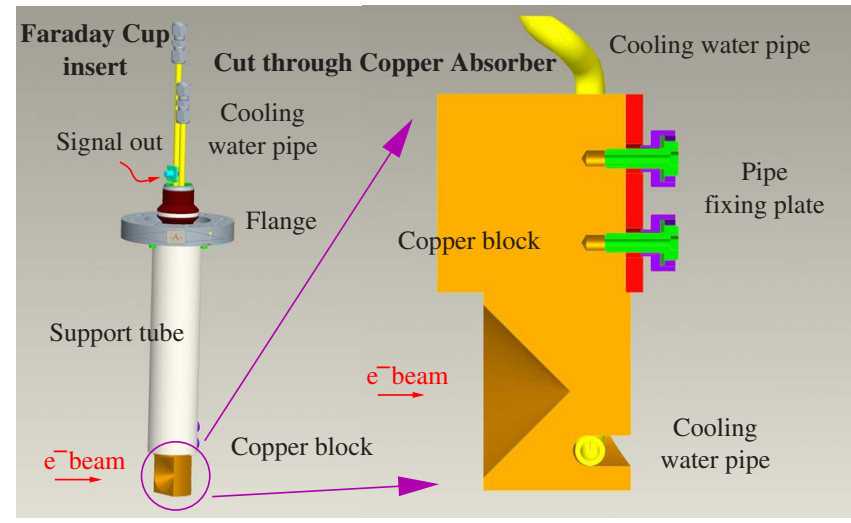

FIG. 2. (Color online) Technical drawing of Faraday cup vacuum insert (left) and detailed cut through the copper absorber block (right). The opening diameter of the funnel-shaped cutout is $36 \mathrm{~mm}$, equivalent to the beam pipe diameter. The minimum depth of the material at the funnel tip is $12 \mathrm{~mm}$.

\section{BEAMLINE OVERVIEW}

A schematic overview of the diagnostics beamline and the SRF gun is given in Fig. 1. The diagnostics beamline consists of

- six viewscreens for transverse beam profile measurements, marked as screens 1-6 in Fig. 1,

- five BPMs (BPM1-BPM5) and four steering coil pairs for orbit control (ST1-ST4),

- two ICTs (ICT 1 and ICT 2) to monitor charge transmission through the beamline,

- one pair of slit masks to analyze the horizontal and vertical beam emittances (EMS),

- one $180^{\circ}$ dipole spectrometer (C-bend) for momentum and momentum spread measurements,

- a Cherenkov monitor and a planned electro-optical sampling (EOS) system to measure the time structure of the electron pulses, and

- three Faraday cups (FCup 1 can be inserted at the beginning of the beamline and two (FCup 2 and FCup3) are included in the beam dumps.

To keep the diagnostics beamline at low pressures (below $10^{-9} \mathrm{mbar}$ ), ion vacuum pumps and ion gauges are mounted below every viewscreen station. For the Cherenkov monitor a differential pumping scheme is applied. Valves are put along the beamline to divide the beamline into different vacuum sectors. The planned EOS system port is currently installed in the ELBE linear accelerator beamline for testing. After completion of the tests it is planned to move the system into the SRF injector beamline.

\section{BEAM CHARGE AND POSITION}

\section{A. Faraday cups}

Faraday cups ${ }^{10}$ and ICTs are used at several positions to measure the total beam intensity. The Faraday cup vacuum insert is shown in Fig. 2. The main part of the system is a block of copper, which is insulated against the electron beam pipe. The block is solid with a funnel-shaped cutout. The relative leakage of electrons was simulated with GEANT (Ref. 
11) and is expected to be $0.4 \%$ for a bunch of $1 \mathrm{nC}$ of $1 \mathrm{~mm}$ size at a $9.5 \mathrm{MeV}$ energy hitting the cup in the center. The Faraday cup is insertable at the location of the first profile monitor port. Two additional Faraday beam dumps are implemented at the dispersive and straight end of the beamline. During commissioning of the beamline, average beam currents as low as $50 \mathrm{nA}$ (at a $100 \mathrm{kHz}$ repetition rate) were measured with a sensitive amperemeter directly connected to the first Faraday cup (FCup1).

\section{B. Integrating current transformer}

Two ICTs (Ref. 12) are used to continuously monitor the bunch charge at two locations, before and in the dispersive arm of the spectrometer dipole. The ICT inductively couples to the beam, requiring an image current bypass around the coil so that the space-charge field of the beam can pass through the windings of the pickup. The electrical conductivity of the beam pipe must be interrupted in the proximity of the ICT; otherwise the wall current will flow through the ICT aperture and cancel the beam current. The beam pipe is interrupted by a ceramic $\mathrm{Al}_{2} \mathrm{O}_{3}$ ring gap sandwiched between two Vacon rings. The ICT together with the piece between the vacuum flanges is housed in a cavity bypass. The transfer impedance of the sensor is expressed in terms of the integral of the output pulse as a function of the input pulse charge. For the ICTs the sensitivity in a $50 \Omega$ load is $5.00 \mathrm{~V} \mathrm{~s} / \mathrm{C}$. The beam charge to output charge ratio in a $50 \Omega$ load is 10:1. At low bunch charges and low repetition rates the signal from the ICTs will be amplified by $22 \mathrm{~dB}$ using lownoise amplifiers. Tests at the ELBE linear accelerator indicate that bunch charges as low as $10 \mathrm{pC}$ can be resolved with $10 \%$ resolution.

\section{Beam position monitors}

The requirements for the beam position measurement are a resolution of better than $100 \mu \mathrm{m}$ for single bunches for beam offsets as far as $5 \mathrm{~mm}$. The readout electronics has to cope with all operation modes. Stripline BPMs as implemented at the ELBE accelerator are used for position monitoring. The striplines have a length of $1 / 4 \lambda_{\mathrm{rf}}$ and are mounted in a compact package. The readout electronics utilizes a logarithmic detector for direct rf to dc conversion and a logarithmic amplifier with a large linear dynamic range of $60 \mathrm{~dB}$. The sensitivity of the BPM system is measured to be $0.8 \mathrm{dBm} / \mathrm{mm}^{13}$

\section{BEAM PROFILE AND EMITTANCE}

\section{A. Beam profile monitor}

The profile monitors are used to image the full beam at several locations along the beamline and to measure the size of the beamlets released by the emittance measurement slit mask. The relative resolution of the profile monitor has to be better than $10 \%$ for full and beamlet imagings. For this purpose $0.2 \mathrm{~mm}$ thick yttrium-aluminum-garnet crystal sheets doped with the visible light scintillator cerium can be inserted into the beam path to produce an image of the transverse charge distribution. This beam image is detected by a charge coupled device (CCD) camera. The screen material has to be robust and ultrahigh vacuum (UHV) compatible because the first screen is located in close proximity to the SRF cavity. To provide for the best image fidelity, the screens are mounted at normal incidence to the electron beam. An aluminum mirror is placed downstream to deflect the fluorescent light out to the camera. Outside the vacuum beam pipe the light is deflected again by a mirror and then focused onto the sensitive area of a CCD camera. The optical focus and magnification can be calibrated by inserting a calibration target at the location of the screen. The minimum object size generated by the crystal screen is dominated by multiple scattering for beam energies below $10 \mathrm{MeV}$. At 2-3 $\mathrm{MeV} / \mathrm{c}$ beam momentum the minimum resolution is around $40 \mu \mathrm{m}$ and at $9.5 \mathrm{MeV} / \mathrm{c}$, the resolution is around $10 \mu \mathrm{m}$. During commissioning, the viewscreens were able to withstand operation conditions with several thousand nanocoulombs per hour without any visible degradation of the beam image. In Fig. 3 the first beam images of the electron beam generated during commissioning are shown.

\section{B. Emittance measurement section}

The electron beam generated in the injector is in all nominal operation modes space-charge dominated. For this reason a double slit-based phase space sampling method ${ }^{14}$ is considered, where an actuator-mounted slit mask is moved perpendicularly across the beam. The purpose of collimating the beam intensity with slits is twofold. The first is to cut low current beam portions out of the high brightness beam. These small beamlets have the same divergence as the original beam with negligible effects due to space charge. The second purpose is to separate the beam into many beamlets, whose intensity distribution at some downstream point can be measured to give the phase space distribution of the beam; the width of each beamlet gives a measure of the width of the transverse momentum distribution at each slit, and the centroid of the beamlets gives the correlated offset of the momentum distribution at each slit. The normalized beam emittance can then be calculated according to

$$
\epsilon_{n}=\beta \gamma \sqrt{\left\langle x^{2}\right\rangle\left\langle x^{\prime 2}\right\rangle-\left\langle x x^{\prime}\right\rangle^{2}} .
$$

Here $\left\langle x^{2}\right\rangle$ and $\left\langle x^{\prime 2}\right\rangle$ are the rms beam size and divergence of the beam, $\left\langle x x^{\prime}\right\rangle^{2}$ is the correlation between size and divergence, and $\beta \gamma$ are the relativistic factors.

The contribution of the slit width toward the beamlet size should be small, but it must be large enough to supply the beamlets with sufficient charge. To meet the requirements of various operation modes, a slit mask with two pairs of slits with 100 and $50 \mu \mathrm{m}$ widths is constructed. For singleshot measurements of the beam emittance, an array with five $100 \mu \mathrm{m}$ slits is also included in the slit mask. The size of the released beamlets will be measured with three viewscreen stations downstream in the diagnostics beamline. The stations are located 0.6, 1.6, and $2.0 \mathrm{~m}$ after the slit mask. The beamlet sizes at these stations vary between 60 and $250 \mu \mathrm{m}$. The mask itself is made of $1.4 \mathrm{~mm}$ thick tungsten, being an optimum between acceptance and background due to Coulomb scattering. The slits were produced by electrodischarge machining the bulk material with a wire. The material is quite brittle, and splinters were found in the slit opening. For 

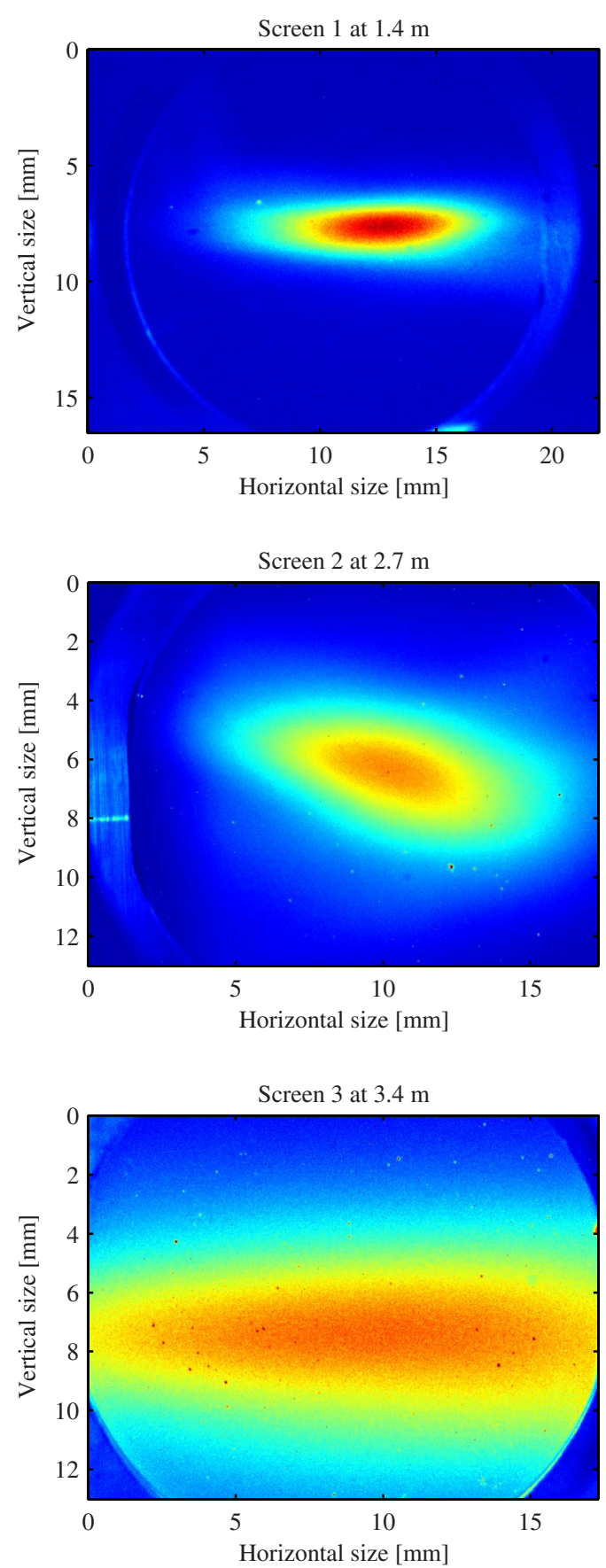

FIG. 3. (Color online) First beam images produced with the electron beam generated by the SRF injector. this reason the $50 \mu \mathrm{m}$ slits were produced by sandwiching two polished half-plates. The complete slit mask is placed inside a carrier (see Fig. 4), which can be moved by an actuator inside the beam path. The alignment of the slits normal to the beam axis can be changed such that the beam passes the full width of the slits without intercepting any material, which would lead to an increase in the transverse beamlet size due to multiple scattering. The tilt of the slit mask can be changed by a movable flexure joint. This second actuator is mounted inside the large actuator moving the mask in and out. The whole package is extremely compact and easy to operate. The size of the individual slits and the spacing of the multislit array were measured by analyzing the Fraunhofer pattern of the slits illuminated by a monochromatic light source. The average slit width for the broad single slits is $d_{\mathrm{sgl}}=85 \pm 2 \mu \mathrm{m}$ and that for the slit array is $d_{\mathrm{arr}}=84 \pm 8 \mu \mathrm{m}$. The spacing of the slit array is $499 \pm 48 \mu \mathrm{m}$. Two slit masks, for the horizontal and vertical planes, are placed inside two separate actuators. Another possibility to measure the transverse emittance is to use the solenoid magnet right after the gun in combination with the first screen for a solenoid-scan-type technique. There the solenoidal focusing is changed and the beam size variation is observed on the first viewscreen.

\section{TEMPORAL PROFILE}

To verify the shape and length of the temporal profile of the electron bunches, two techniques with picosecond time resolution will be used. Inside the Cherenkov monitor ${ }^{15,16}$ the electron bunches pass a thin sheet of radiator. The radiator emits a Cherenkov radiation pulse with the same time structure as the electron bunch. A streak camera can be used to measure the shape and length of this radiation pulse with picosecond resolution. This technique is destructive to the electron bunches as the beam size increases during the passage through the radiator. The EOS diagnostic technique ${ }^{17}$ inspects the electric field copropagating with the electron bunch and enables nondestructive single-shot measurement with subpicosecond resolution.

\section{A. Cherenkov monitor}

The first temporal profile monitor is located after the spectrometer magnet in a straight direction. The Cherenkov process is characterized by the Cherenkov angle $\cos \theta_{C}$

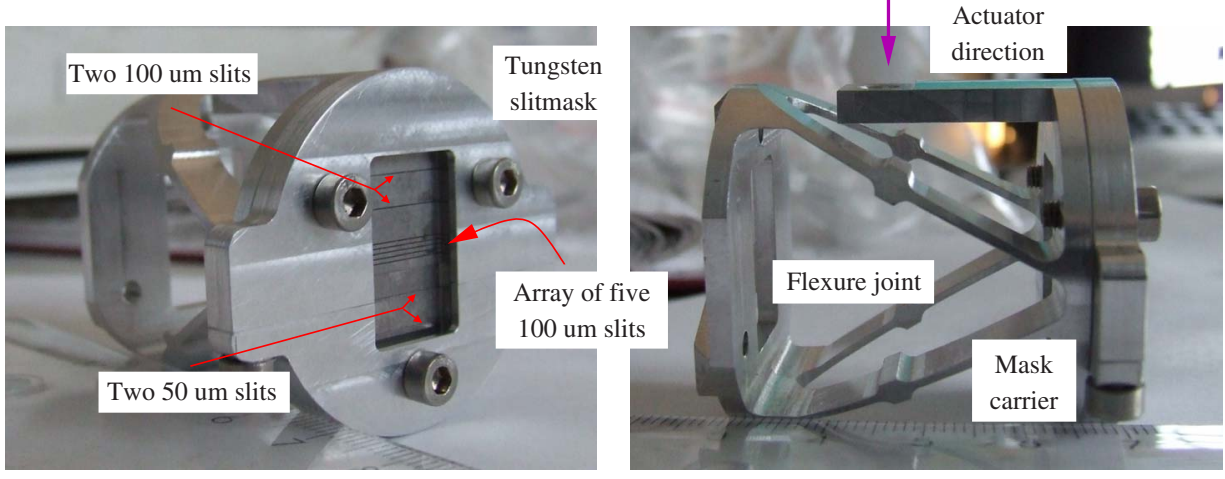

FIG. 4. (Color online) EMS slit mask inside the carrier, which can be tilted with respect to the beam by a flexure joint. 


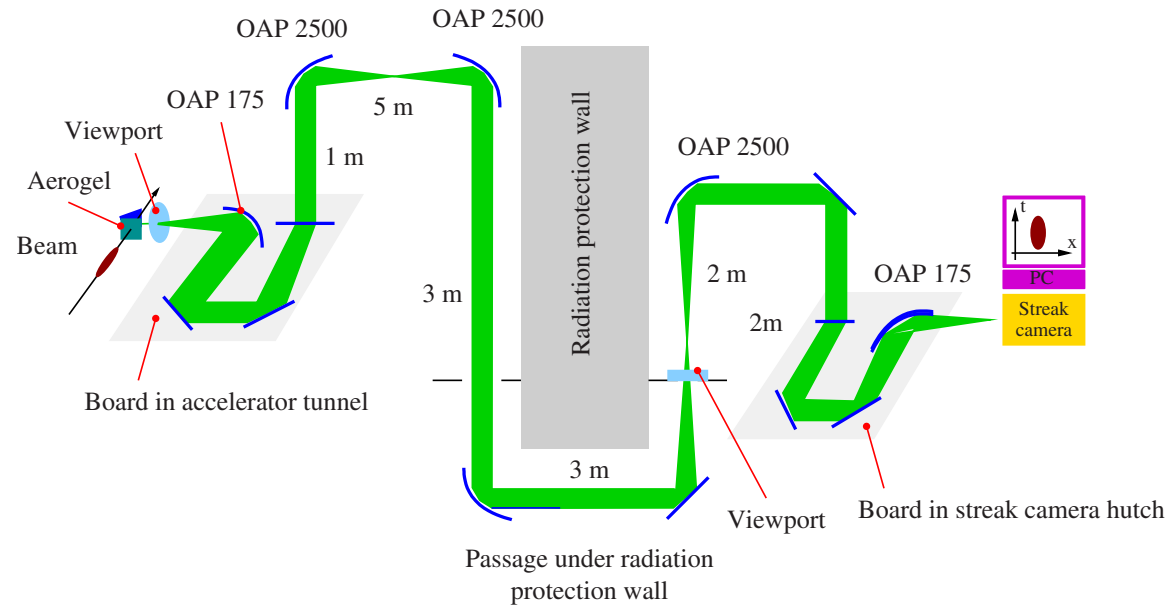

FIG. 5. (Color online) Schematic of the Cherenkov light beam path. The light is guided and focused by plane and off-axis parabolic mirrors. The parabolic mirrors have focal lengths of $2500 \mathrm{~mm}$ off-axis parabolic (OAP of 2500) and $175 \mathrm{~mm}$ (OAP of 175). The ring of the Cherenkov light at the source is imaged with a double Gaussian relay telescope on the entrance of the Streak camera.

$=1 / \beta n$ (where $\beta$ is the relativistic velocity factor of the electrons and $n$ the refractive index of the radiator), under which a radiation cone is emitted. The number of emitted photons per pathlength in a bandwidth between wavelengths $\lambda_{1}$ and $\lambda_{2}$ is

$$
\frac{d N_{\gamma}}{d x}=2 \pi \alpha z^{2} \sin ^{2} \theta_{C} \frac{\lambda_{2}-\lambda_{1}}{\lambda_{1} \lambda_{2}},
$$

where $z$ is the charge in elementary charge units and $\alpha$ the fine-structure constant. ${ }^{18}$ Silica aerogel plates of small dimensions with refractive indices of $n=1.008$ and 1.028 are available $^{19}$ and considered as a Cherenkov radiator. The threshold energy for $n=1.008$ is $4.1 \mathrm{MeV}$ and that for $n$ $=1.028$ is $2.2 \mathrm{MeV}$. The refractive index of the material was measured with samples in a parallel plate setup. A test plate of thickness $d$ was put perpendicular into the light path of a laser beam. The plate was rotated and the resulting parallel shift of the laser beam measured. From the measured parallel shift versus rotation angle data, the refractive index could be retrieved. Results from measurements under atmospheric pressure can be used to estimate the refractive index of the material under UHV pressure. ${ }^{20}$ The thickness of the plate was chosen to be $6 \mathrm{~mm}$ to balance the amount of emitted photons with the time resolution due to dispersion. ${ }^{21}$ The Cherenkov light is then transported with mirrors to the streak camera $^{22}$ over a distance of roughly $20 \mathrm{~m}$. The Cherenkov light exits the radiator with a large divergence; therefore a relay imaging with focusing elements is necessary to achieve a high photon collection efficiency. Using lenses for this can cause dispersion related pulse broadening of the short photon pulse. For setups with lenses, pulse broadening by a factor of 2.5 was measured for bunches of 20 ps FWHM length. ${ }^{23}$ Therefore only reflective optical elements, plane and off-axis parabolic mirrors, were considered for the light transport. In Fig. 5 the light transport is sketched. The setup was simulated with the ray-tracing code RAY. ${ }^{24}$ The pulse is broadened due to dispersion in the light beamline by 2.2 ps for wavelengths between 300 and $600 \mathrm{~nm}$, caused to almost equal parts by the two viewports and the air in the light beamline. These effects can be minimized by using bandpass filters.

\section{B. Laser pulse measurements}

First tests with the streak camera were performed to check the laser pulse length and the synchronization of the streak camera to the master oscillator. For the direct measurement an optical transport line with mirrors and beamsplitters was set up to transport the laser light from the laser hutch to the neighboring streak camera hutch. Synchronization between the laser and streak camera is achieved by a $250 \mathrm{MHz}$ phase-locked loop synthesizer driven by a $13 \mathrm{MHz}$ reference signal from the laser itself. The synchronization accuracy between the laser and streak camera was measured to be better than $2 \mathrm{ps}$. The laser oscillator delivers pulses in the infrared $(1053 \mathrm{~nm})$, which are amplified and finally converted into two second harmonic generators to green (526 $\mathrm{nm}$ ) and finally ultraviolet $(263 \mathrm{~nm})$. During the data taking, the pump current of the photodiodes of the laser amplifier was changed and the average power and pulse length of the output pulses were measured. For measurement of the green pulses, the second conversion stage was removed. The average laser power was measured with a powermeter and the pulse length with the streak camera, as described above. For each pump current setting, the light intensity entering the streak camera was regulated, the green pulses by lowreflectivity optical flats and neutral density filters and the UV only with low-reflectivity flats. In Fig. 6 the results for these measurements are shown. For each data point five streak images were taken. Each image was analyzed by applying a Gaussian fit to the time-projection axis data. The error bars for the pulse length data reflect the standard deviation from these five measurements. The systematic uncertainty is 2 ps for this particular streak camera model. For the green pulses the average output power increases with increasing pump current. Above a pump current of $15.5 \mathrm{~mA}$, a sudden drop in laser intensity was observed. The laser intensity recovered soon, but due to time constraints the data point for this pump current could not be taken again. For the UV output pulses, the sequence started at high pump currents. Here also the laser intensity dropped, this time at the data point for a pump current below $18.5 \mathrm{~mA}$. The intensity for the measurement 

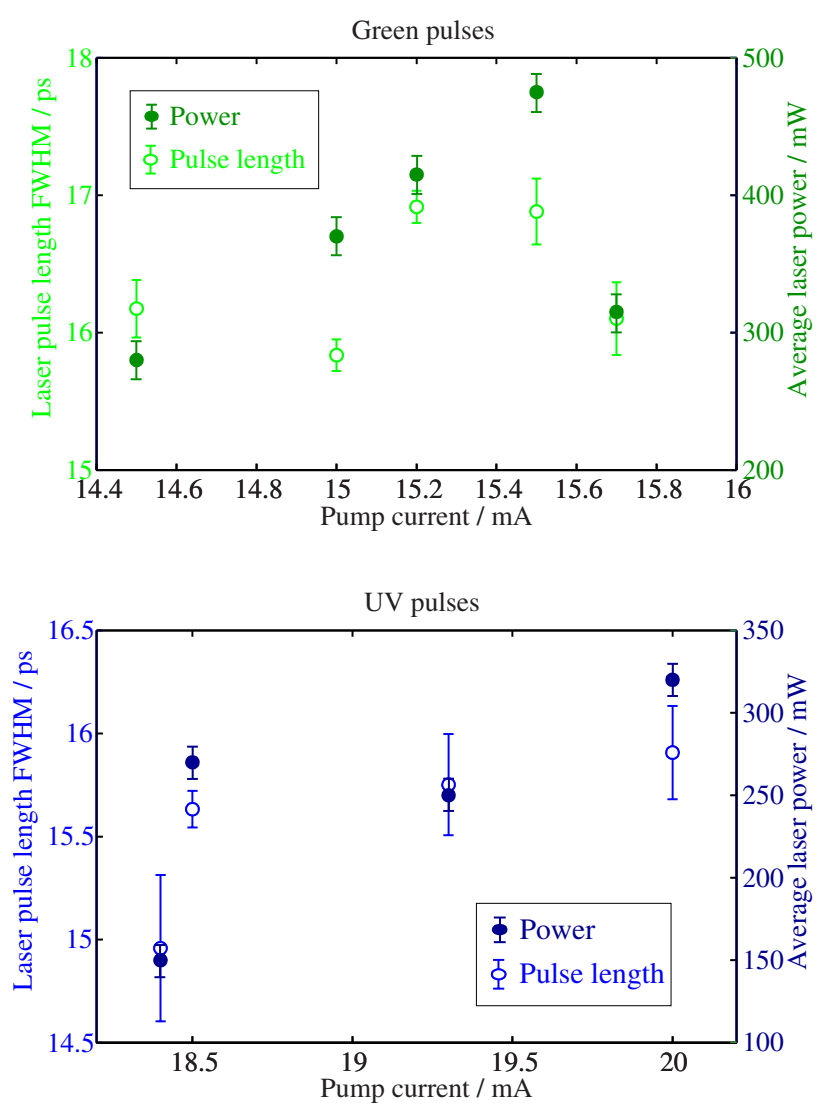

FIG. 6. (Color online) Results from laser pulse length measurements for the green (green and open green points) and the UV (blue and open blue points) output of the laser for different pump currents.

on the streak camera was consequently very low, leading to a small signal to noise ratio and thus to an underestimation of the pulse length. It is planned to do the measurements again. The pulse length of the electron bunches after extraction out of the cathode as measured with a phase scan is $\Delta z_{e}=18.1 \pm 7.3$ ps FWHM.

\section{Electro-optical sampling}

EOS is a method based on the detection of the Coulomb fields accompanying the electron bunch. With this technique short laser pulses are used to sample the time structure of the Coulomb field. In the EOS setup, the EO crystal is mounted in the beam pipe with the surface perpendicular to the electron beam orbit and the edge of the crystal a few millimeters away from it. When the electron bunch passes the crystal, the Coulomb field enters the crystal and travels through it, inducing birefringence. Due to this birefringence, a laser pulse acquires a polarization change, which can be measured with a $\lambda / 4$ plate and a Glan-Taylor prism in a balanced detector setup (see Fig. 7). By changing the optical delay for each electron bunch, a different position along the longitudinal axis of the Coulomb field can be detected. The setup is currently not installed in the diagnostics beamline but it is installed in the ELBE linear accelerator for performance tests.

\section{MOMENTUM AND MOMENTUM SPREAD}

The momentum distribution of the electron beam will be measured with a $180^{\circ}$ dipole magnet spectrometer. Simula-

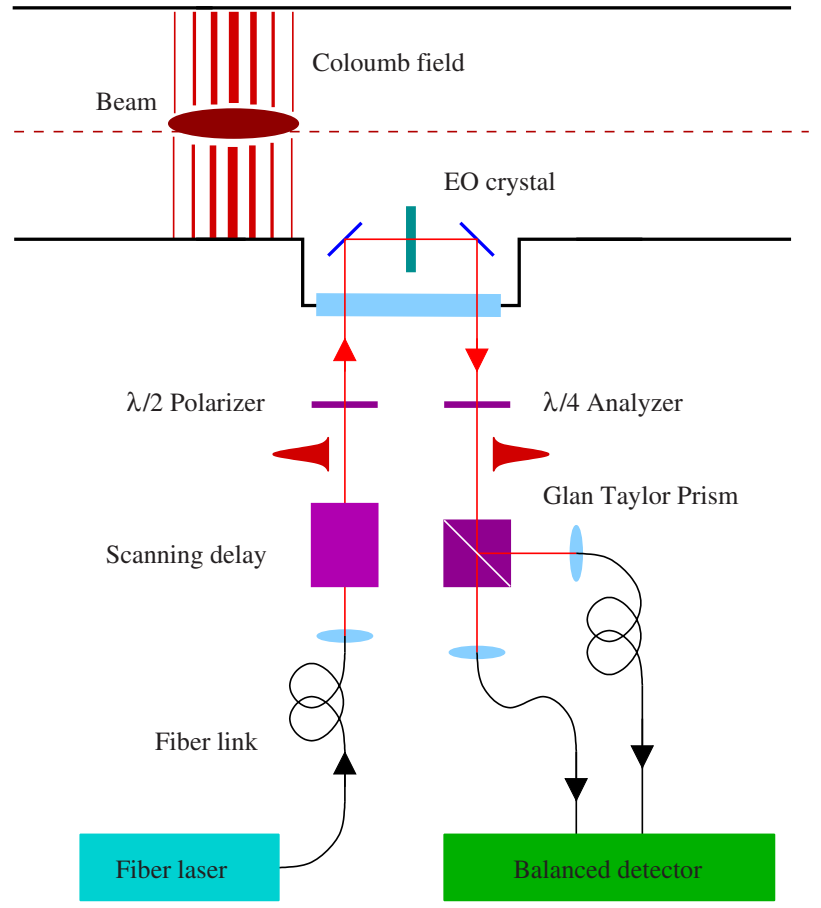

FIG. 7. (Color online) Sketch of the electro-optical sampling setup.

tions with the $77 \mathrm{pC}$ bunch charge operation mode indicate that a normalized emittance of $\epsilon_{n}=0.6 \mathrm{~mm}$ mrad together with an uncorrelated momentum spread of $\Delta p=36 \mathrm{keV} / \mathrm{c}$ can be reached, setting a limit for the desired resolution of the device. The spectrometer is an H-type dipole with a bending radius of $\rho=200 \mathrm{~mm}$ and a gap height of $g$ $=40 \mathrm{~mm}$. The field map of the magnet was measured with a Hall probe on a movable stage. By moving the dipole and the Hall probe, a two-dimensional map of the field distribution was measured. The relative homogeneity of the field between the pole shoes was measured to be $2.5 \times 10^{-4}$, which is below the value specified for this magnet of $10^{-3}$, which enables measurement of the momentum spread with an accuracy of better than $0.1 \%$. A $180^{\circ}$ dipole magnet was chosen in order to unfold the contribution of the emittance and Twiss parameter to the beam size measured in the dispersive arm. An electron in the dispersive arm with fractional momentum $\Delta p / p$ will have a transverse position on screen SC5 (see Fig. 1) according to

$$
x_{S C 5}=R_{11} x_{o}+R_{12} x_{o}^{\prime}+R_{16} \frac{\Delta p}{p},
$$

where $x_{o}$ and $x_{o}^{\prime}$ denote the electron beam position and divergence upstream the dipole and $R_{i j}$ are the elements of the transfer matrix ${ }^{25}$ a between the initial location and the screen. The deconvolution can be done by putting a second screen in the nonbend plane in a location with the same $\left|R_{11}\right|$ and $\left|R_{12}\right|$ as for the bend plane. Such a screen is placed at the position of the Cherenkov monitor of the beamline (SC4 in Fig. 1). Simulating the setup with the particle tracking code ASTRA ${ }^{26}$ indicates that a momentum spread as low as $\Delta p$ $=6.3 \mathrm{keV} / \mathrm{c}$ at $10 \mathrm{MeV}$ beam energy and $1 \mathrm{nC}$ bunch charge can be resolved using the setup and the deconvolution technique. 


\section{SUMMARY AND OUTLOOK}

The diagnostics beamline currently under construction plays a vital role in the commissioning and successful running of the SRF injector. Results from first beam tests at low bunch charge indicate the efficiency of the individual devices.

\section{ACKNOWLEDGMENTS}

The authors would like to thank the engineers and technicians from BESSY, FZD, and the Max Born Institute who contributed to the SRF injector project. The authors are grateful for the assistance received by DESY Hamburg and SLAC. The project is supported by the German Federal Ministry of Education and Research Grant No. 05 ES4BR1/8.

${ }^{1}$ The BESSY Soft X-Ray Free Electron Laser, TDR BESSY March 2004, edited by D. Krämer, E. Jaeschke, and W. Eberhardt (BESSY, Berlin, 2004).

${ }^{2}$ P. Michel, H. Buttig, F. Gabriel, M. Helm, U. Lehnert, C. Schneider, R. Schurig, W. Seidel, D. Stehr, J. Teichert et al., The 28th International Free Electron Laser Conference (FEL 2006), Berlin, Germany, 27 August-1 September 2006 (unpublished).

${ }^{3}$ D. H. Dowell, S. Z. Bethel, and K. D. Friddell, Nucl. Instrum. Methods Phys. Res. A 356, 167 (1995).

${ }^{4}$ S. S. Kurennoy, D. C. Nguyen, D. L. Schrage, R. L. Wood, V. Christina, J. Rathke, T. Schultheiss, and L. M. Young, Proceedings of the Particle Accelerator Conference (PAC 05), Knoxville, TN, 16-20 May 2005, p. 2866.

${ }^{5}$ K. Goldammer, Diploma thesis, Technical University Berlin, 2004.

${ }^{6} \mathrm{X}$. Chang and J. Kewisch, Proceedings of the Particle Accelerator Conference (PAC 07), Albuquerque, NM, 2007 (unpublished), p. 3187.
${ }^{7}$ D. Janssen and V. Volkov, Nucl. Instrum. Methods Phys. Res. A 452, 34 (2000).

${ }^{8}$ A. Arnold, H. Buettig, D. Janssen, T. Kamps, G. Klemz, W. D. Lehmann, U. Lehnert, D. Lipka, F. Marhauser, P. Michel et al., Nucl. Instrum. Methods Phys. Res. A 577, 440 (2007).

${ }^{9}$ I. Will, G. Klemz, F. Staufenbiel, and J. Teichert, The 28th International Free Electron Laser Conference (FEL 2006), Berlin, Germany, 27 August-1 September 2006 (unpublished).

${ }^{10}$ K. L. Brown and G. W. Tautfest, Rev. Sci. Instrum. 27, 696 (1956).

${ }^{11}$ S. Agostinelli, J. Allison, K. Amako, J. Apostolakis, H. Araujo, P. Arce, M. Asai, D. Axen, S. Banerjee, G. Barrand et al., Nucl. Instrum. Methods Phys. Res. A 506, 250 (2003).

${ }^{12}$ Bergoz Instrumentation, 2006

${ }^{13}$ P. Evtushenko, Ph.D. thesis, Technical University Dresden, 2004.

${ }^{14}$ J. Rosenzweig, G. Travish, UCLA PBPL Note No. 362, 1994.

${ }^{15}$ T. Watanabe, J. Sugahara, T. Yoshimatsu, S. Sasaki, Y. Sugiyama, K. Ishi, Y. Shibata, Y. Kondo, K. Yoshii, T. Ueda et al., Nucl. Instrum. Methods Phys. Res. A 480, 315 (2002).

${ }^{16}$ M. E. Conde, W. Gai, R. Konecny, X. Li, J. Power, P. Schoessow, and N. Barov, Phys. Rev. ST Accel. Beams 1, 041302 (1998).

${ }^{17}$ I. Wilke, A. M. MacLeod, W. A. Gillespie, G. Berden, G. M. H. Knippels, and A. F. G. van der Meer, Phys. Rev. Lett. 88, 124801 (2002).

${ }^{18}$ C. Grupen, Particle Detectors (Cambridge University Press, Cambridge, 1996).

${ }^{19}$ A. Yu Barnyakov, M. Yu. Barnyakov, J. Bähr, T. Bellunato, K. I. Beloborodov, V. S. Bobrovnikov, A. R. Buzykaev, M. Calvi, A. F. Danilyuk et al., Nucl. Instrum. Methods Phys. Res. A 553, 125 (2005).

${ }^{20}$ D. Richter and D. Lipka, Nucl. Instrum. Methods Phys. Res. A 513, 635 (2003).

${ }^{21}$ J. Bahr, V. Dzhordzhadze, D. Lipka, A. Onuchin, and F. Stephan, Nucl. Instrum. Methods Phys. Res. A 538, 597 (2005).

${ }^{22}$ Hamamatsu Photonics, 2003.

${ }^{23}$ D. Lipka, Ph.D. thesis, Humboldt University, 2004.

${ }^{24}$ F. Schäfers, BESSY Technical Report No. TB-202, 1996.

${ }^{25}$ K. L. Brown, SLAC Report SLAC-R-075, 1968.

${ }^{26} \mathrm{~K}$. Floettman, ASTRA, A space-charge tracking algorithm. 